\title{
La dieta hipocalórica asociada a sibutramina fue eficaz para reducir el peso y mantenerlo en forma sostenida
}

Long-term Maintenance of Weight Loss after a Very-Low-Calorie Diet: A Randomized Blinded Trial of the Efficacy and Tolerability of sibutramine. Apfelbaum M, Vague P, Ziglier 0, et al. Am J Med. 1999;106:179-184.

\section{Objetivo}

Evaluar si el uso de sibutramina asociada a una dieta hipocalórica es efectiva para reducir el peso corporal y mantenerlo en forma sostenida.

\section{Diseño}

Ensayo clínico aleatorizado y controlado doble ciego con un seguimiento de 15 meses.

\section{Lugar}

Doce centros médicos franceses especializados en obesidad y endocrinología.

\section{Pacientes}

Hombres y mujeres entre 18 y 55 años, con BMI> 30. Se excluyó a los obesos de causas endocrinológicas, diabéticos tipo 1 o tipo 2 insulinizados o mal controlados, (glucemia 7,8 mmol/l), aquellos con tensión arterial diastólica $>100 \mathrm{~mm} / \mathrm{hg}$, a los que habían estado sometidos a una dieta hipocalórica en los 6 meses previos al estudio, a los depresivos, y a los que estuviesen cumpliendo con una terapia farmacológica que pudiera interferir los resultados del estudio.

\section{Intervención}

Inicialmente se administró a todos los pacientes seleccionados una dieta de muy bajas calorías, (220-800 kcal), durante un mes. Luego de este periodo, se randomizó solo a los que habían perdido como mínimo $6 \mathrm{~kg}$ de peso, a recibir $10 \mathrm{mg}$ de sibutramina por día o placebo durante 12 meses. Ambos grupos recibían una dieta con un contenido de
20-30 \% menos de calorías, en comparación a la dieta que cumplían antes de ingresar al estudio.

\section{Medición de resultados principales}

Se utilizó como resultado principal a la pérdida de peso corporal expresada en $\mathrm{kg}$, y la proporción de pacientes que mantuvieron el 100\%, $50 \%, 25 \%$ del peso perdido luego de la dieta de muy bajas calorías.

\section{Resultados principales}

Se incluyeron 81 pacientes en el grupo sibutramina y 78 en el grupo placebo. El análisis fue por intención de tratar. En el momento de la randomización no había diferencias significativas de peso entre ambos grupos. Al cumplir los 12 meses de seguimiento el grupo sibutramina cambió $-5.2(+/-7.5) \mathrm{kg}$, y el grupo control $+0.5(+/-5.7) \mathrm{kg}$, $(p=0.004)$. La proporción de pacientes que mantuvo el 100,50 y $25 \%$ de la pérdida del peso luego de la dieta inicial de muy bajas calorías fueron el 75, 95 y $96 \%$ en el grupo sibutramina y del 42,73 y $80 \%$ en el grupo control. Al mes de haber terminado el tratamiento los cambios en el peso fueron: $1.6(+/-$ 1.5) $\mathrm{kg}$ para el grupo sibutramina y $1.0(+/-2.1) \mathrm{kg}$ para el grupo control. A los 3 meses los cambios fueron de $4.3(+/-3.1) \mathrm{kg}$ y $2.3(+/-2.9) \mathrm{kg}$ para el grupo sibutramina y control respectivamente.

\section{Conclusiones}

La asociación de subitramina con una dieta hipocalórica fue más efectiva que el placebo para reducir el peso corporal y mantenerlo durante un año. Al finalizar el período de randomización ambos grupos recuperaron peso, en especial el de sibutramina, lo que demuestra la importancia de la medicación para mantener el peso.

\section{COMENTARIO}

La sibutramina es un inhibidor de la recaptación de serotonina y noradrenalina, utilizado para el tratamiento de la obesidad. Su eficacia fue demostrada en varios trabajos que como este, no superan los 15 meses de seguimiento. ${ }^{1-3}$ Ninguno informa que pasó con los pacientes años después, dato importante ya que la obesidad es una enfermedad crónica que se presenta tanto como factor de riesgo cardiovascular, y se asocia a un mayor riesgo de muerte por enfermedad coronaria; como a mayor prevalencia de diabetes, dislipemias, gota, osteoartritis, depresión, etc. El presente trabajo no fue originalmente diseñado para evaluar el comportamiento a largo plazo. Al ser la obesidad una enfermedad crónica el tratamiento debe ser de por vida, haciendo hincapié en la modificación de los hábitos alimentarios y en la actividad física.

De recibir tratamiento farmacológico, sea sibutramina, orlistat, u otro, al suspenderlo los pacientes tienden a retornar al peso previo a su administración. Es por eso que hay que pensar muy bien a quien administrar estos tratamientos aun no probados a largo plazo y con un importante costo para el paciente. ${ }^{4}$

La validez externa* de este trabajo se ve muy limitada ya que el grupo de participantes tiene características muy distintas a la de la mayoría de los obesos: era una población sin ninguna otra morbilidad, con mucha voluntad para adherirse al tratamiento durante un período prolongado, condiciones que se alejan de la realidad del consultorio. Por otro lado, hubo una importante pérdida de pacientes durante el seguimiento. De utilizarse, la sibutramina, se debe utilizar con mucha precaución en los pacientes con hipertensión diastólica y no se recomienda en los que tienen hipertensión no controlada o enfermedad cardiovascular concomitante, ya que la droga aumenta el riesgo de isquemia cardíaca.

El trabajo describe también, en coincidencia con otros estudios, los beneficios de la droga sobre el perfil lipídico, incrementando los niveles de HDL y disminuyendo los de triglicéridos y ácido úrico, pero su importancia clínica no está aclarada.

Es evidente que aún queda un largo camino por recorrer en el manejo farmacológico de esta enfermedad. El médico de atención primaria no debe olvidar que el tratamiento de la obesidad es complejo y muy difícil de resolver, y que debe ser encarado en forma multidisciplinaria a largo plazo.

*Ver Glosario

Unidad de Medicina Familiar y Preventiva. Hospital Italiano de Buenos Aires.

\section{Referencias}

1. Luque CA, Rey JA, Sibutramine: a serotonin-norepinephrine reuptake-inhibitor for the treatment of obesity. Ann Pharmacother 1999 Sep-33(9):968-78.

2. Mc Neely W, Goa KL, Sibutramine. A review of its contribution to the management of obesity. Drugs 1998 Dec;56(6):1093-124.

3. Bray GA, Ryan DH, A double-blind randomized placebo-controlled trial of sibutramine. Obes Res 1996 May;4(3):263-70.

4. Aronne LJ, Modern medical management of obesity: the role of pharmaceutical intervention. J Am Diet Ass 1998 Oct;98(10 suppl 2):S23-6. 